\title{
Antibiotic Utilization Pattern in Tertiary Hospital Intensive Care Unit in West Java, Indonesia
}

\author{
Taradharani Wikantiananda, ${ }^{1}$ Adi Imam Tjahjadi, ${ }^{2}$ Reza Widianto Sudjud ${ }^{3}$ \\ ${ }^{1}$ Faculty of Medicine Universitas Padjadjaran, Indonesia \\ ${ }^{2}$ Department of Biomedical Science, Faculty of Medicine Universitas Padjadjaran, Indonesia \\ ${ }^{3}$ Department of Anesthesiology and Intensive Care Faculty of Medicine Universitas Padjadjaran/Dr. Hasan \\ Sadikin General Hospital Bandung, Indonesia
}

Abstract Objective: To explore the pattern of antibiotic utilization in intensive care unit (ICU).

Methods: This was a retrospective study on patients in ICUs of a Tertiary
Hospital Intensive Care Unit in West Java, Indonesia. Subjects were recruited
using the total sampling method during the period of January to June 2016.
Data collected were processed using the Anatomical Therapeutic Chemical
(ATC) Classification/Defined Daily Dose (DDD) system as the international
measurement standard for analysis and comparison from the World Health
Organization (WHO).

Results: Fifty-seven medical records were collected with the total antibiotics used of 295.72 DDD/100 bed-days. Levofloxacin, meropenem, ceftriaxone, ceftazidime, and metronidazole were the five most frequently used antibiotics with $143.18,49.88,30.62,19.74$, and 16.99 DDD/100 bed-days, respectively.

Received:

February 19, 2019

Revised:

September 08, 2019

Accepted:

October 19, 2019
Conclusions: Ceftriaxone is the predominantly used antibiotics that is provided to $54.39 \%$ of the patients while levofloxacin is the antibiotics with the highest number of doses used with a total of 143.18 DDD/100 bed-days.

Keywords: Antibiotics, DDD, intensive care unit

pISSN: 2302-1381; eISSN: 2338-4506; http://doi.org/10.15850/ijihs.v7n2.1633

IJIHS. 2019;7(2):81-7

\section{Introduction}

Intensive care unit (ICU) is a dedicated area to support the lives of critical patients in health care facilities. ICU plays an important role in treating patients with severe infectious diseases; however, it should also be noted that ICU stays may trigger several complications and even death. ${ }^{1}$ Nosocomial infections can occur in the intensive care units due to invasive actions, such as the installation of mechanical ventilation, urinary catheters, and various other invasive procedures. ${ }^{2}$

Antibiotic resistance continues to increase

Correspondence:

Taradharani Wikantiananda,

Faculty of Medicine Universitas Padjadjaran

Jalan Raya Bandung Sumedang Km. 21

Jatinangor, Sumedang, Indonesia

e-mail: t.wikantiananda@gmail.com as inappropriate administration of antibiotics sustains. Currently, antibiotic resistance has become one of the biggest global health threats. WHO has declared antibiotic resistance as one of the three most important problems for public health in this century. ${ }^{3}$ A 2011 WHO survey in Indonesia demonstrates that the antibiotics consumption contributes $22-41 \%$ of the total number of drugs distributed in the country. ${ }^{4}$ A study on quantitative antibiotic use in University of Zurich Hospital that antibiotic use in ICU ranks third among the top used drugs in the hospital with 451.80 Defined Daily Dose (DDD)/100 patients.

A study of antimicrobial resistance in two indonesian hospitals in 2000-2004 discovered that $30-80 \%$ of the antibiotics were not used as indicated. ${ }^{5}$ Changes in bacterial resistance patterns occur due to different therapeutic patterns in each period of time, especially because of the inappropriate administration 
Table 1 Patient Characteristics

\begin{tabular}{lcccc}
\hline \multicolumn{1}{c}{ Patient Characteristics } & $\begin{array}{c}\text { Male } \\
(\mathbf{n = 2 3})\end{array}$ & $\begin{array}{c}\text { Female } \\
(\mathbf{n = 3 4 )}\end{array}$ & $(\mathbf{n = 5 7 )}$ & \% \\
\hline 0-5 years & 0 & 0 & 0 & 0 \\
6-11 years & 0 & 0 & 0 & 0 \\
12-25 years & 3 & 4 & 7 & 12 \\
26-45 years & 4 & 12 & 16 & 28 \\
46-65 years & 10 & 13 & 23 & 40 \\
$>$ 65 years & 6 & 5 & 11 & 19 \\
\hline
\end{tabular}

or inappropriate type of antibiotics given. ${ }^{6}$ Antibiotic resistance increases due to improper use of antibiotics as a consequence of the lack of a standardized antibiotic use guideline and supervision. ${ }^{7}$ This study aimed to explore antibiotic use in the ICU to identify its utilization pattern. It is expected that the result of this study can be used as a reference and consideration when evaluating which antibiotics should be use and how to provide antibiotic treatment more effectively.

\section{Methods}

This was a retrospective descriptive study conducted in August 2018 on patients treated in the intensive care unit of a tertiary hospital in West Java, Indonesia, during the period of January to June 2016. The ethical clearance for this study has been obtained from the Ethics Committee for Health Research, Faculty of Medicine, Universitas Padjadjaran (No. 218/ UN6.KEP/EC/2018), and Ethics Committee of Dr. Hasan Sadikin, Bandung General Hospital (No. LB.02.01/X.2.2.1/8353/2018).

Data used in this study were secondary data collected from the medical records by applying the total sampling method. Fifty- seven patient's medical records were used in the study. The inclusion criteria used was all medical records of ICU patients who were given antibiotics while medical records with incomplete and missing data were excluded.

Variables collected were patient's length of stayin theintensive care unit, type of antibiotics, antibiotic dose, and antibiotic resistance test. Data were then analyzed using the Anatomical Therapeutic Chemical (ATC) classification/ Defined Daily Dose (DDD) system from the WHO, which is an international measurement standard for analyzing and comparing drug use. The DDD was divided by the patient days and presented as DDD/100 bed-days. The ATC code and WHO DDD antibiotic numbers are available at https://www.whocc.no/atc_ddd_ index/.

\section{Results}

There were 86 patients treated in the intensive care unit during the study period but the medical records of 13 patients were not stored at the medical record unit of the hospital. Of these, 57 medical records met the inclusion criteria while 14 medical records

Table 2 Number of Antibiotic Type Prescribed per patients

\begin{tabular}{lcc}
\hline \multicolumn{1}{c}{ Number of Antibiotic Type } & \multicolumn{2}{c}{ Patient (N=57) } \\
\cline { 2 - 3 } & $\mathbf{n}$ & $\mathbf{\%}$ \\
\hline type of antibiotic & 16 & $28.07 \%$ \\
2 types of antibiotics & 14 & $24.56 \%$ \\
3 types of antibiotics & 16 & $28.07 \%$ \\
4 types of antibiotics & 7 & $12.28 \%$ \\
5 types of antibiotics & 3 & $5.26 \%$ \\
6 types of antibiotics & 1 & $1.75 \%$ \\
\hline
\end{tabular}


Table 3 Frequency of Individual Antibiotics Prescribed

\begin{tabular}{lcc}
\hline \multirow{2}{*}{\multicolumn{1}{c}{ Antibiotic }} & \multicolumn{2}{c}{ Patient (n=57) } \\
\cline { 2 - 3 } Amikacin & $\mathbf{n}$ & $\mathbf{\%}$ \\
\hline Cefepime & 4 & 7.02 \\
Cefotaxime & 1 & 1.75 \\
Ceftazidime & 3 & 5.26 \\
Ceftriaxone & 13 & 22.81 \\
Cefazolin & 31 & 54.39 \\
Ciprofloxacin & 5 & 8.77 \\
Erythromycin & 1 & 1.75 \\
Gentamicin & 1 & 1.75 \\
Levofloxacin & 1 & 1.75 \\
Linezolid & 24 & 42.11 \\
Meropenem & 1 & 1.75 \\
Metronidazole & 27 & 47.37 \\
Piperacillin/tazobactam & 1 & 31.58 \\
Teicoplanin & 1 & 1.75 \\
Tetracycline & 1 & 1.75 \\
Tigecycline & 4 & 1.75 \\
Vancomycin & 4 & 2.84 \\
\hline
\end{tabular}

were excluded because the medical records were not available in the ICU.

Of the 57 patients who met the inclusion criteria, female comprised the majority of the patients with 34 patients $(60 \%)$, while the number of male patients were 23 (40\%). Most patients treated in the intensive care unit belongs to the age category of 46-65 years. The patients' total length of stay in the ICU during the study period was 418 days. The distribution of subject characteristics is presented in Table 1.

There were 18 types of antibiotics used for these 57 patients. On average, 2.47 types of antibiotics were given to each patient during the study period. Resistance tests were performed in 17 patients (29.82\%). The frequency of each type of antibiotics is presented in Table 3.

The total number of antibiotic used in the ICU was 295.72 DDD/100 bed-days. Table 4 shows that the three most commonly used antibiotics were levofloxacin, ceftriaxone, and meropenem with the total use of $143.18,49.88$, and $30.62 \mathrm{DDD} / 100$ bed-days, respectively.
Table 5 shows that 131 antibiotic regimen were used in the ICU and $57.25 \%$ of the therapy included a combination of antibiotics.

In total, there were 48 types of antibiotics that were used, both in combination and as an individual antibiotic (Table 6).

\section{Discussion}

On average, 2.47 antibiotics were administered to each patient in the intensive care unit during the period of January-June 2016. This number is comparable to the number presented by a study in North India which found that the average antibiotic administration per patient was 3.36. However, this number is low when compared to the finding in other similar studies in East India with an average antibiotic administration of 10.5.,

A study conducted in 2016 in South India found that the most frequently used antibiotic is ceftriaxone, which is given to $22.77 \%$ of patients. ${ }^{10}$ This is consisted with the finding in this study as the most frequently used antibiotics were, in a descending order, ceftriaxone (54.39\%), meropenem $(47.37 \%)$, levofloxacin (42.11\%), metronidazole (31.58\%), and ceftazidime (22.81\%).

The total number of antibiotic uses in this study reached 295.72 DDD/100 bed-days with levofloxacin, ceftriaxone, meropenem, ceftazidime, and metronidazole as the five most frequently used antibiotics. This is relatively high when compared to the finding of a similar study in the University of Zurich Hospital with a total number of antibiotic use of $125.88 \mathrm{DDD} / 100$ bed-days. This is also high when compared to a study in South India in 2014 with a total use of antibiotics of 148.97 DDD/100 bed-days. . $^{10,11}$

The relatively higher use of antibiotics in this study probably links to the fact that other previous studies were performed in 2006 and 2014 while the use of antibiotics tends to increase from year to year. Similar results are demonstrated by a study in Brazil where antibiotic use increases from 83.8 DDD/100 bed-days in 1990 to $124.6 \mathrm{DDD} / 100$ beddays in $1996 .{ }^{10,12}$ The higher use of antibiotics does not always reflect effective treatments and reduction in patient mortality rate. A retrospective study in Germany found that the overall mortality rate and resistance level fall when antibiotic consumption drops from 67.1 to $51.0 \mathrm{DDD} / 100$ bed-days. ${ }^{13}$

There is a fear that the relatively high antibiotic use in the ICU of the tertiary hospital where this study conducted will 
Table 4 Antibiotic Use Pattern

\begin{tabular}{lcccc}
\hline Type of Antibiotic & ATC Code & $\begin{array}{c}\text { WHO DDD } \\
\text { (g) }\end{array}$ & $\begin{array}{c}\text { Total DDD } \\
\text { Unit Used }\end{array}$ & $\begin{array}{c}\text { DDD/100 } \\
\text { Bed-days }\end{array}$ \\
\hline Amikacin & J01GB06 & 1 & 19.00 & 4.55 \\
Cefepime & J01DE01 & 2 & 12.00 & 2.87 \\
Cefotaxime & J01DD01 & 4 & 8.25 & 2.97 \\
Ceftazidime & J01DD02 & 4 & 82.50 & 19.74 \\
Ceftriaxone & J01DD04 & 2 & 128.00 & 30.62 \\
Cefazolin & J01DB04 & 3 & 16.00 & 3.83 \\
Ciprofloxacin & J01MA02 & 0.5 & 14.40 & 3.44 \\
Erythromycin & J01FA01 & 1 & 7.50 & 1.79 \\
Gentamicin & J01GB03 & 0.24 & 10.67 & 2.55 \\
Levofloxacin & J01MA12 & 0.5 & 598.50 & 143.18 \\
Linezolid & J01XX08 & 1.2 & 7.00 & 1.67 \\
Meropenem & J01DH02 & 2 & 208.50 & 49.88 \\
Metronidazole & J01XD01 & 1.5 & 71.00 & 16.99 \\
Piperacillin/Tazobactam & J01CA12 & 14 & 0.03 & 0.01 \\
Teicoplanin & J01XA02 & 0.4 & 10.00 & 2.39 \\
Tetracycline & J01AA07 & 1 & 8.00 & 1.91 \\
Tigecycline & J01AA12 & 0.1 & 19.00 & 4.55 \\
Vancomycin & J01XA01 & 2 & 15.75 & 3.77 \\
\hline
\end{tabular}

Table 5 Characteristics of Antibiotic Use in ICU

\begin{tabular}{lcc}
\hline \multicolumn{1}{c}{ Characteristics } & \multicolumn{2}{c}{ Regiment (N=131) } \\
& $\mathbf{n}$ & \% \\
\hline Antibiotic use & & \\
Monotherapy & 56 & 42.75 \\
Combination & 75 & 57.25 \\
Number of antibiotic type used & & \\
Single Antibiotic & 56 & 42.75 \\
Combination of 2 Antibiotics & 54 & 41.22 \\
Combination of >2 Antibiotics & 21 & 16.03 \\
Duration of administration & & \\
< 2 Days & 46 & 80.70 \\
2-3 Days & 44 & 59.65 \\
4-7 Days & 34 & 80.70 \\
> 7 Days & 46 & 8.77 \\
\hline
\end{tabular}


Taradharani Wikantiananda, Adi Imam Tjahjadi, et al.

Table 6 Frequency of Antibiotic Prescription

\begin{tabular}{|c|c|c|}
\hline \multirow{2}{*}{ Antibiotics } & \multicolumn{2}{|c|}{ Prescription $(\mathrm{N}=131)$} \\
\hline & $\mathbf{N}$ & $\%$ \\
\hline Amikacin & 2 & $1.53 \%$ \\
\hline Cefepime & 1 & $0.76 \%$ \\
\hline Ceftazidime & 3 & $2.29 \%$ \\
\hline Ceftriaxone & 22 & $16.79 \%$ \\
\hline Cephazolin & 5 & $3.82 \%$ \\
\hline Levofloxacin & 6 & $4.58 \%$ \\
\hline Meropenem & 13 & $9.92 \%$ \\
\hline Metronidazole & 1 & $0.76 \%$ \\
\hline Tigecyclin & 1 & $0.76 \%$ \\
\hline Vancomycin & 2 & $1.53 \%$ \\
\hline Amikacin-levofloxacin & 1 & $0.76 \%$ \\
\hline Amikacin-meropenem & 2 & $1.53 \%$ \\
\hline Amikacin-tigecyclin & 1 & $0.76 \%$ \\
\hline Amikacin-vancomycin & 1 & $0.76 \%$ \\
\hline Cefotaxime-ceftazidime & 1 & $0.76 \%$ \\
\hline Cefotaxime-levofloxacin & 1 & $0.76 \%$ \\
\hline Cefotaxime-metronidazole & 1 & $0.76 \%$ \\
\hline Ceftazidime-levofloxacin & 7 & $5.34 \%$ \\
\hline Ceftazidime-meropenem & 1 & $0.76 \%$ \\
\hline Ceftazidime-metronidazole & 2 & $1.53 \%$ \\
\hline Ceftriaxone-gentamycin & 1 & $0.76 \%$ \\
\hline Ceftriaxone-levofloxacin & 4 & $3.05 \%$ \\
\hline Ceftriaxone-meropenem & 2 & $1.53 \%$ \\
\hline Ceftriaxone-metronidazole & 7 & $5.34 \%$ \\
\hline Ceftriaxone-vancomycin & 1 & $0.76 \%$ \\
\hline Ciprofloxacin-metronidazole & 1 & $0.76 \%$ \\
\hline Erythromycin-tigecyclin & 1 & $0.76 \%$ \\
\hline Levofloxacin-meropenem & 11 & $8.40 \%$ \\
\hline Meropenem-metronidazole & 5 & $3.82 \%$ \\
\hline Meropenem-vancomycin & 1 & $0.76 \%$ \\
\hline Teicoplanin-tigecyclin & 1 & $0.76 \%$ \\
\hline Tigecyclin-vancomycin & 1 & $0.76 \%$ \\
\hline Amikacin-levofloxacin-meropenem & 1 & $0.76 \%$ \\
\hline Ceftazidime- cefepime-levofloxacin & 1 & $0.76 \%$ \\
\hline Ceftazidime-ceftriaxone-levofloxacin & 3 & $2.29 \%$ \\
\hline Ceftazidime-levofloxacin-meropenem & 1 & $0.76 \%$ \\
\hline Ceftazidime-levofloxacin-tigecyclin & 1 & $0.76 \%$ \\
\hline Ceftriaxone-gentamycin-levofloxacin & 1 & $0.76 \%$ \\
\hline
\end{tabular}

International Journal of Integrated Health Sciences. 2019;7(2):81-7 


\begin{tabular}{llc} 
Ceftriaxone-levofloxacin-meropenem & 1 & $0.76 \%$ \\
Ceftriaxone-meropenem-metronidazole & 4 & $3.05 \%$ \\
Cephazolin-ciprofloxacin-metronidazole & 1 & $0.76 \%$ \\
Ciprofloxacin-meropenem-metronidazole & 1 & $0.76 \%$ \\
Levofloxacin-meropenem-metronidazole & 1 & $0.76 \%$ \\
Levofloxacin-meropenem-tetracyclin & 1 & $0.76 \%$ \\
Linezolide-meropenem-metronidazole & 1 & $0.76 \%$ \\
Meropenem-metronidazole-vancomycin & 1 & $0.76 \%$ \\
Teicoplanin-piperacillin/tazobactam-tigecyclin & 1 & $0.76 \%$ \\
Cephazolin-levofloxacin-meropenem-metronidazole & 1 & $0.76 \%$ \\
\hline
\end{tabular}

increase antibiotic resistance and reduce the effectiveness of antibiotic treatment. In early 2018, a study in the United States found that the risk of individuals contracting antibioticresistantbacteria increases with the proportion of patients treated with antibiotics. ${ }^{14}$ A study in Lebanon at the end of 2017 also revealed that antibiotic use in hospitals are generally associated with an increased vulnerability of a hospital to antibiotic resistance. ${ }^{15}$

The data in this study showed that there were 131 antibiotic prescriptions given and $57.25 \%$ of them consisted of an antibiotic combination. The most common single antibiotic used was ceftriaxone $(16.79 \%)$, and the most common antibiotic combination used was the combination of levofloxacin and meropenem (8.40\%). This antibiotic combination is considered necessary to increase the effectiveness of therapy by expanding the spectrum of therapy or to anticipate antibiotic resistance. ${ }^{16}$

In conclusion, the use of antibiotics in the intensive care unit of the tertiary hospital in this study is considered high with a total of 295.72 DDD/100 bed-days when compared to other similar studies. The most frequently used of antibiotics is ceftriaxone, which is used in $54.39 \%$ of patients. Whereas in number, the most widely used antibiotic is levofloxacin with a total of $143.18 \mathrm{DDD} / 100$ bed-days.

This study has many limitations, such as the retrospective nature of the study, the limited time (six months) for observing the antibiotic use pattern, and that the data used are only based on medical records. However, it can be seen that the types and regimens for antibiotic administration are very diverse. The use of antibiotics must be evaluated and a policy of antibiotic administration should be applied to reduce the excessive use of antibiotics.

\section{References}

1. Kleinpell R, Blosser S, Goldner J, Birriel B, Fowler CS, Byrum D, et al. ICU admission, discharge, and triage guidelines: A framework to enhance clinical operations, development of institutional policies, and further research. Crit Care Med. 2016;1553-1602.

2. Kouchak F, Askarian M. Nosocomial infections: the definition criteria. Iran J Med Sci. 2012;37(2):72-3.

3. WHO. Antimicrobial resistance, Global report on surveillance. 2014.

4. Holloway K. Pharmaceuticals in Health Care Delivery. Mission Rep. 2011;(February):1-38.

5. Kementrian Kesehatan RI. Penggunaan Antibiotik Bijak dan Rasional Kurangi Beban Penyakit Infeksi. 2015;

6. Wahjono H, Kristina TN. Auditing peta medan kuman dan antibiogram sebagai educatedguess penanganan penyakit infeksi. Media Med Indones. 2012;46(36):6-11.

7. Leung E, Weil DE, Raviglione M, Nakatani H. The WHO policy package to combat antimicrobial resistance. Bull World Health Organ. 2011;89(5):390-2.

8. Mahajan B, Kaushal S, Chopra SC. A drug utilization study of antimicrobial agents (AMAs) in the Intensive Care Units (ICUs) at Medical College Hospital of North India. 2013;15(3):129-32.

9. Patanaik SK, Pattanayak C, Prasad A, Chauhan AS. Drug utilization pattern in an intensive care unit setting in Eastern India. International Journal of Basic \& Clinical Pharmacology. 2015;4(6):1136-41.

10. Anand N, Nagendra Nayak IM, Advaitha 
MV, Thaikattil NJ, Kantanavar KA, Anand S. Antimicrobial agents' utilization and cost pattern in an Intensive Care Unit of a Teaching Hospital in South India. Indian J Crit Care Med. 2016;20(5):274-9.

11. Kuster SP, Ruef C, Ledergerber B, Hintermann A, Deplazes C, Neuber L, et al. Quantitative antibiotic use in hospitals: Comparison of measurements, literature review, and recommendations for a standard of reporting. Urban \& Vogel. 2008;36(6):549-59.

12. de Castro M, Pilger D, Ferreira M, Kopittke L, De. Trends in antimicrobial utilization in a University Hospital, 1990-1996. Rev Saude Publica. 2002;36(553-8).

13. Scholze K, Wenke M, Schierholz R, Groß U, Bader 0, Zimmermann 0, et al. The Reduction in. 2015;2011(December 2013).

14. Almagor J, Temkin E, Benenson I, Fallach N, Carmeli Y. The impact of antibiotic use on transmission of resistant bacteria in hospitals: Insights from an agent-based model. PLoS One. 2018;13(5)(e0197111).

15. Wang A, Daneman N, Tan C, Brownstein J, MacFadden D. Evaluating the relationship between hospital antibiotic use and resistance in common nosocomial pathogens. Infect Control Hosp Epidemiol. 2017;38(12):145763.

16. Ahmed A, Azim A, Gunjar M, Baronia A. Current concepts in combination antibiotic therapy for critically ill patients. Indian J Crit Care Med. 2014;18(5)(310-4). 